\title{
An exploratory investigation of online and offline social behaviour among digital natives
}

\author{
Amy Broughton \\ Department of Information Science, \\ Stellenbosch University \\ Stellenbosch, South Africa \\ Michael Nieuwoudt \\ Department of Information Science, \\ Stellenbosch University \\ Stellenbosch, South Africa
}

\author{
Mila Daly \\ Department of Information Science, \\ Stellenbosch University \\ Stellenbosch, South Africa \\ Daniel B. le Roux \\ Department of Information Science, \\ Stellenbosch University \\ Stellenbosch, South Africa \\ dbleroux@sun.ac.za
}

\author{
Nickel-Jon Marx \\ Department of Information Science, \\ Stellenbosch University \\ Stellenbosch, South Africa \\ Douglas A. Parry ${ }^{*}$ \\ Department of Information Science, \\ Stellenbosch University \\ Stellenbosch, South Africa \\ dougaparry@sun.ac.za
}

\begin{abstract}
In response to calls for IS researchers to investigate how digital natives are using information and communication technologies to shape their interpersonal interactions, an exploratory, surveybased study was conducted to investigate patterns of online social behaviour on two popular SNSs (Facebook and Instagram) and, on this basis, compare their online and offline social behaviour. To capture online social behaviour a novel typology involving three high-level interactions - producing, consuming, and reacting- was proposed. The investigation found that, among digital natives, Instagram is used more frequently than Facebook, with consumptive behaviour on Instagram occurring most frequently, and productive behaviour on Facebook occurring least frequently. While online social behaviour was found to positively relate to offline socialisation, personality traits were found to account for a larger proportion of the variance in offline social behaviour than online actions. The findings are of particular relevance to those seeking to understand associations between behaviour online and behaviour offline. Moreover, the typology introduced is likely to be useful in a variety of contexts.
\end{abstract}

\section{CCS CONCEPTS}

- Applied computing $\rightarrow$ Sociology; Law, social and behavioral sciences; Psychology; • Human-centered computing $\rightarrow$ Ubiquitous and mobile computing.

\section{KEYWORDS}

Social Behaviour, Online Behaviour, Offline Behaviour, Facebook, Instagram

\section{ACM Reference Format:}

Amy Broughton, Mila Daly, Nickel-Jon Marx, Michael Nieuwoudt, Daniel B. le Roux, and Douglas A. Parry. 2019. An exploratory investigation of online and offline social behaviour among digital natives. In Conference of the South African Institute of Computer Scientists and Information Technologists 2019

SAICSIT '19, September 17-18, 2019, Skukuza, South Africa

(C) 2019 Association for Computing Machinery.

This is the author's version of the work. It is posted here for your personal use. Not for redistribution. The definitive Version of Record was published in Conference of the South African Institute of Computer Scientists and Information Technologists 2019 (SAICSIT '19), September 17-18, 2019, Skukuza, South Africa, https://doi.org/10.1145/3351108.3351146.
(SAICSIT'19), September 17-18, 2019, Skukuza, South Africa. ACM, New York, NY, USA, 10 pages. https://doi.org/10.1145/3351108.3351146

\section{INTRODUCTION}

People are understood to be inherently social with strong desires to forge and maintain relations through interactions with acquaintances, peers, friends, and other connections [5]. Such behaviour is generally associated with enhanced psycho-social well-being and positive affective outcomes [35]. Therefore, it is not surprising that we are producing evermore sophisticated tools to augment our social interactions with one another. Through a variety of online social networking services (SNSs) - platforms enabling the creation and connection of profiles, the exchange of information, and the management of social connections [9]- many of our information and communications technologies enable continuous social interaction. SNSs are now used by a majority of adolescents and adults alike [31]. Facebook and Instagram, in particular, attract a widevariety of users across demographic and socio-economic spectra, with a majority using these services daily.

Social behaviour refers to interactions between individuals or groups of individuals. Two concepts are of particular importance: agency, which refers to the individual capacity to act independently and structure, the factors that influence the enactment of agency $[19,36]$. While there is much debate about the primacy of agency or structure, through their interaction, individuals act in relation to their environments. Specifically, the structure of a situation directs actions in accordance with, firstly, the norms of the situation, secondly, the available artefacts in the situation and, thirdly, other individuals within the situation. It can be argued that, as artefacts of communication, digital media, and SNSs in particular, can structure social interactions. It is not unwarranted, therefore, to presume that SNSs may hold profound implications for the nature of social interaction in general.

The present study is situated within the agenda for Information Systems (IS) research proposed by Vodanovich et al. [45]. These authors call attention to the changing nature of information technologies and suggest that, for digital natives ${ }^{1}$, use of the Internet and mobile digital technologies is now an indispensable part of

\footnotetext{
${ }^{1}$ A category of users defined by Prensky [33] as those who have grown up in a digital world. Digital immigrants, on the other hand, are those users who started interacting with information systems in the course of their adult lives.
} 
their lives. Along with laptops and mobile phones Vodanovich et al. [45, p. 711] provide various SNSs (e.g., Facebook, Twitter) as examples of, what they term, ubiquitous information systems (UIS) -the hardware as well as the "people, processes, information, and communication systems and technologies [...] that are interconnected and interwoven into the very fabric of our lives through ubiquitous networks" (p. 713). Noting that IS research has primarily focused on organisational contexts, these authors propose that, given the increasing blur between organisational and non-organisational contexts, the use of UISs for personal and professional purposes, and the changing nature of the future workforce (i.e, the transition from digital immigrants to digital natives), IS researchers should endeavour to study how members of this generation are interacting with such digital technologies.

Given the centrality of UISs in life today, the need to understand digital natives' use of UISs, and indications of associations between behaviour online and behaviour offline [28, 35, 47], the primary objective of this study is to describe patterns of online social behaviour exhibited by digital natives (specifically university students) on two popular SNSs (Facebook and Instagram) and, on this basis, compare their online and offline social behaviour. Additionally, given associations between personality traits and behaviour online [2, 23], associations between the well-established Big Five model of personality and online social behaviour are examined.

This study was conducted within an exploratory framework, with no a priori hypotheses preregistered. Consequently, while valuable insights are provided, caution in interpreting the outcomes is encouraged. As argued by Wagenmakers et al. [46] exploratory research forms an essential component of an academic domain. Emphasis is placed on identifying and describing interesting aspects of the data, determining which tentative findings are of particular interest for future study and, importantly, proposing ways in which future research can confirm or dis-confirm these exploratory results. To this end, extending from the literature reviewed in the next section, in Section 3, specific research questions are posed to guide the study. Following this, the details of the survey methodology adopted to address these questions are outlined. Thereafter, the results are presented before a discussion thereof is provided.

\section{LITERATURE REVIEW}

To contextualise the investigation key characteristics of SNSs are briefly described. Next, behaviour on SNSs is considered culminating in the description of a typology of SNS behaviour. Finally, previous studies investigating associations between online and offline behaviour are described.

\subsection{Social Networking Services}

SNSs enable users to "construct a public or semi-public profile within a bounded system, articulate a list of other users with whom they share a connection, and view and traverse their list of connections and those made by others within the system" [9, p. 211]. Users are, accordingly, provided with toolsets to "facilitate engagement in social interactions" [47, p. 5]. While the goals of communication and social interaction may exist in both online and offline social networks, as Oh et al. [26] note, the affordances of online SNSs enable such goals to be enacted in unique and distinct ways. Different SNSs have different properties which partly determine the affordances of the user-platform relationship and, additionally, how users can interact with each other. Facebook, for instance, enables undirected relationships between users [12], while Instagram involves a network of directed relationships. Referring to Facebook, Smock et al. [38] contend that the service can be considered as a collection of tools utilized in different ways to meet different needs. Instagram, has been described as an online mobile photo/video capturing and sharing service [22]. While similar features exist, Facebook presents a different set of tools to users.

Today, engagement with various SNSs is one of the most common uses of the World Wide Web, especially among digital natives [31]. Remaining the most popular platform, over a billion users across age-groups access Facebook on a daily basis [25]. Among young adults (who are likely digital natives), in addition to Facebook, a majority use Instagram daily. In South Africa the trends are similar. Specifically, while no data is available on daily usage and despite high-costs of mobile data and other barriers to Internet connectivity, 21-million South Africans currently have Facebook accounts and 6.6 million have Instagram accounts [48].

\subsection{A Typology of Behaviour on SNSs}

While many studies consider media use by assessing the frequency of use, a growing number of researchers have recognised the need to assess particular aspects of media use and investigate how individuals engage with SNSs $[29,47]$.

In an early typology of behaviour on SNSs and online forums Nielsen [24] proposed a 90-9-1 rule to describe the nature of active participation on such platforms. $90 \%$ of users are lurkers who do not contribute but simply consume information. The next group of users, the intermittent contributors (9\%), occasionally contribute but more generally consume content contributed by others. The heavy contributors $(1 \%)$ are the active users who, collectively, account for a majority of the activity on a platform. While this typology has received much attention, it has not been empirically assessed and, given the considerable developments in SNSs over the subsequent 13 years, whether it still holds remains uncertain.

Burke et al. [11] characterised two distinct forms of SNS use: active directed communication and passive undirected communication. Direct communication includes platform-interactions between a user and another user involving direct engagement between the two (e.g., commenting, direct-messaging). In contrast, passive communication refers to the monitoring of content not specifically directed at a user (e.g., status broadcasts, posts, shares) through reading a feed of such content. For Facebook, Gerson et al. [18] provide evidence indicating that passive communication is now the norm on the platform.

Brandtzaeg and Heim [10] developed a typology of SNS users based on participation level (intensity of use) and participation mode (objective of use). A user may be either low or high in their level of participation but can simultaneously have a recreational or informational mode of use. In a survey-based assessment ( $n=5$ 233) Brandtzaeg and Heim [10] identified five clusters of responses and, on this basis, proposed five user types reflecting different combinations of use-intensity and participation objectives. Active 
users were described as those who frequently engage in almost all kinds of participation activities, while lurkers are those who generally engage in passive use. Sporadics are those who used SNSs only occasionally and tended toward an informational mode of use. Socialisers engaged actively in recreational use, while debaters tended toward an informational mode of use.

More recently, in a similar manner to Burke et al. [11], Wenninger et al. [47] propose that a distinction between targeted (reciprocityevoking) and non-targeted (non-reciprocity-evoking) interaction modes on SNSs is required. In focusing on a particular recipient, targeted actions (e.g., commenting, liking) are more likely to initiate a response than non-targeted actions (e.g., browsing, sharing) [6] and, as a consequence, positively effect social bonding and connectedness. In contrast, non-directed interactions, browsing for instance, have been associated with perceptions of disconnectedness and exclusion from social groups [39,44].

Given indications that the mode of interaction is an important determinant of associations between use of SNSs and psycho-social well-being [4, 13, 47], it is evident that a typology of SNS behaviour is necessary for the present study. Consequently, it is proposed that, across the platform-specific tools and affordances, three high-level interactions emerge: producing, consuming, and reacting. Productive behaviour refers to actions on a SNS that lead to the creation of new content (e.g., posting statuses, posts, or stories). In contrast, consumptive behaviour, as a passive form of SNS use, involves the consumption of content produced by other users. Reactive behaviour, while active, refers to actions which extend directly from the actions of other users (e.g., liking, sharing, or commenting). Both reactive and productive are directed and reciprocity-evoking, while consumption is not. This typology is used to simplify the range of online social behaviour into a number of distinct, meaningful categories. While previous typologies have sought to classify users, it is argued that, in classifying actions, a more complete understanding of online social behaviour can be achieved. Moreover, in presenting an abstract typology of SNS behaviour, the specific affordances of various platforms are implicitly incorporated, while not emphasising the unique features or action possibilities of any one platform. Additionally, the typology ignores the users' motivations or objectives for engaging in a particular action. While such factors are certainly important for understanding online behaviour, they are not within the scope of the present investigation.

\subsection{Associations Between Online and Offline Social Behaviour}

It has been argued that, through use of various SNSs, individuals can foster a sense of social connectedness and maintain their social relationships [11]. In contrast, others have argued that such services atrophy social connection and diminish psycho-social well-being [41]. Turkle [41] contends that connecting with others over the Internet only provides a perception of connection or friendship and, despite this interaction, social bonding and connectedness suffer. Supporting this assertion, it has been shown that there is no association between the size of an individual's online social network and their offline social network [32].
While there are similarities between offline social interaction and online social interaction -the exchange of information, homophily, and network management for instance- important distinctions exist between the two. Such differences relate, primarily, to the characteristics and affordances of SNSs as Internet mediated platforms. In particular, SNSs have properties of non-locality and non-temporality -interactions can take place regardless of time and place. This is not the case for offline interaction, which requires temporal and environmental overlap. Furthermore, the affordances of SNSs allow for passive interaction without immediate reciprocal interaction $[10,11]$. In contrast, offline social interaction is typically characterised by reciprocal sharing in which increasingly intimate information is exchanged, strengthening social bonds [27].

Over the preceding decade, researchers from a variety of disciplines have considered possible associations between use of SNSs and various indicators of offline social behaviour. Despite the aforementioned differences, such studies have produced varied findings -research has shown both positive and negative associations between online and offline social behaviour. Providing support for positive associations, Gilbert and Karahalios [20] found that SNS activity was a strong predictor of relationship strength. Other studies have shown that use of SNSs increases perceptions of social connectedness and strengthens social bonds [1, 21, 37]. Such studies and others have, simultaneously, found that increased use of SNSs can also be associated with diminished social connectivity and social bonding, and increased perceptions of loneliness [8, 35, 40]. Ryan et al. [35] note that this effect has come to be termed the displacement hypothesis [42]. Time on SNSs replaces time that would have ordinarily been spent engaging in offline social experiences.

As noted previously, the manner in which an individual uses a SNS has been shown to have differential effects on offline social behaviour [4, 13, 47]. Consequently, it may be that certain modes of interaction promote connectedness, while others do not. Burke et al. [11] found that, after controlling for directed communication, frequent passive consumption on SNSs is associated with reduced bridging social capital. For directed communication (productive or reactive behaviour), Burke et al. [11] found positive associations with bonding social capital. Supporting this, Deters and Mehl [15] found, through an online experiment, that those who were required to post more frequently on a SNS indicated reduced loneliness due to increases in feelings of connectedness. In addition to the mode of use, evidence suggests that the individual user characteristics (e.g., personality, gender) can influence, firstly, the nature of behaviour online and, secondly, the nature of psycho-social outcomes associated with such behaviour [17, 22, 37, 43]. Extroversion, in particular, has been associated with increased use of SNSs [23].

\section{METHOD}

A survey, distributed as a web-based self-administered questionnaire to a student-sample, was developed to address the study objective. To guide this investigation the following research questions were formulated:

- RQ1: With specific reference to the proposed typology of SNS behaviour, what is the pattern of behaviour exhibited by digital natives on Facebook (RQ1.1) and Instagram (RQ1.2)? 
- RQ2: How does online social behaviour differ between the two platforms?

- RQ3: What are the associations between online social behaviour and offline social behaviour?

- RQ4: What are the associations between the Big Five Personality traits and online social behaviour?

- RQ5: Does online social behaviour account for a significant amount of variability in offline social behaviour over and above that accounted for by personality traits?

\subsection{Survey Design}

To address the research questions a web-based survey consisting of four sections was designed to collect data at a large, residential, South African university. The first section of the survey elicited demographic and socioeconomic information. Items included: age, gender, number of years at university, monthly social spending budget, and funding source. Following this, the survey included the Mini International Personality Item Pool (Mini-IPIP) [16] Five Factor Model. $^{2}$ This is a 20-item short version of the 50 item IPIP five factor model commonly used to assess individuals' propensities for each of the Big Five personality dimensions (extroversion, agreeableness, conscientiousness, neurotocism, and openness). Responses were recorded using 5-point Likert scales ranging from Strongly Disagree (1) to Strongly Agree (5), with four items presented for each personality dimension. For each trait relevant items are summed to produce a score ranging from four to 20 , with a higher score representing a greater prominence of the trait. ${ }^{3}$ This scale has been shown to be internally consistent, reliable and commensurate in terms of correlational patterns with longer measures of personality [16]. Moreover, while differences have been shown, a number of studies have confirmed the criterion validity, and five factor structure of the Mini-IPIP [3, 14]. For the current sample the subscales were generally internally consistent (extroversion: $\alpha=0.82$; agreeableness: $\alpha=0.77$; conscientiousness: $\alpha=0.61$; neuroticism: $\alpha=0.71$; openness: $\alpha=0.67$ ).

The third section concerned online social behaviour. For each of the SNSs considered, after eliciting daily use time, the same nine items were presented. These nine items were grouped into three categories (production, consumption, and reaction). In the production category the following items were presented: "I often post statuses or posts on [platform]"; "I often post stories on [platform]"; and "I often post live stories on [platform]". In the consumption category the following items were presented: "I spend long periods of time scrolling through my [platform] news feed or viewing others' profiles "; "I often view stories others have posted on [platform]"; and "I often view live stories others have posted on [platform]". Finally, in the reactive category the following three items were presented: "I often like others' [platform] posts and statuses"; "I often comment on [platform] posts and statuses"; and "I often share others' posts and statuses on [platform]". Responses were provided

\footnotetext{
${ }^{2}$ The relevant items are in the public domain and are presented by Donnellan et al. [16] in the original publication.

${ }^{3}$ To calculate a score for extroversion, items 6 and 16 were reverse coded and summed with items 1 and 11. Similarly, for agreeableness, items 7 and 17 were reverse coded and summed to items 2 and 12 . For conscientiousness, items 8 and 18 were reverse coded and summed with items 3 and 13. For neuroticism, items 9 and 19 were reverse coded and summed with items 4 and 14. Finally, for openness to experience, items 10 , 15 and 20 were reverse coded and summed with item 5.
}

through seven-point Likert scales with response options: never (0), a couple of times a year (1), once a month (2), once a week (3), a couple of times a week (4), daily (5), and multiple times a day (6). In the analysis the items were considered individually and, for each dimension of SNS behaviour, summed together to form a separate combined scale.

The final section of the survey presented four items relating to offline social behaviour, with responses provided through the same seven-point Likert scales as the nine online behaviour items. The first item required respondents to indicate how often they socialise in a group of fewer than five people. The second concerned the frequency of socialisation in a group of more than five people, the third the frequency of socialisation with family members, and the fourth considered the frequency of socialisation with peers outside of a work or academic environment.

\subsection{Data Collection}

A sample of students (undergraduate or postgraduate) who, at the time of data collection, had an active account on at least one of the two SNSs considered in the study, was targeted for data collection. To gather such a sample, the questionnaire was distributed through an online survey hosted on the Checkbox platform for a pre-specified, two week data collection period. Respondents were invited through A4 posters placed around the main campus of the institution, announcements in four undergraduate classes (spanning first year to third year, with 2500 students from three faculties Economic and Management Sciences, Arts and Social Sciences, and Natural Sciences), and through distribution on institution-specific groups or pages on various SNSs. Prior to distribution, the survey was piloted with a group of 10 students from the target population. This enabled the questionnaire to be adapted for ambiguity, response-inaccuracy and legibility. Additionally, ethical and institutional clearance were obtained from the relevant authorities.

\section{ANALYSIS AND RESULTS}

The analysis commences with a description of the sample considered. Thereafter, descriptive analyses are presented for online social behaviour before associations between online and offline social behaviour are considered. This is followed by an analysis of associations between personality traits and online social behaviour. Finally, the section concludes with an analysis of the proportion of variance in offline social behaviour explained by online social behaviour, after accounting for personality traits. In all of the analyses an a priori alpha level of 0.05 was adopted and, unless indicated, all assumptions of the statistical procedures were satisfied.

\subsection{Sample Description}

Over the two-week data collection period 260 complete and 234 incomplete responses were received (completion rate: $52.63 \%$ ). Incomplete responses were removed for all analyses. The final sample ( $n=260)$ included 159 females $(61.15 \%)$, 99 males (38.08\%) and two respondents $(0.77 \%)$ who identified with other gender descriptors. Overall, the mean age of the sample was 21.10 years $(S D=2.21)$, with $78.08 \%$ enrolled for undergraduate degrees and $21.92 \%$ for postgraduate degrees. The largest proportion of respondents were in their first year of study $(n=94 ; 36.15 \%)$, while $30(11.54 \%)$ were 
Table 1: Average daily SNS use duration.

\begin{tabular}{lrrrr}
\hline Daily Use (hours) & \multicolumn{2}{c}{ Facebook } & \multicolumn{2}{c}{ Instagram } \\
\hline & $\mathrm{n}$ & $\%$ & $\mathrm{n}$ & $\%$ \\
One & 160 & 72.07 & 91 & 42.33 \\
Two & 42 & 18.92 & 69 & 32.09 \\
Three & 12 & 5.41 & 36 & 16.74 \\
Four & 4 & 1.80 & 11 & 5.12 \\
More than five & 4 & 1.80 & 8 & 3.72 \\
\hline
\end{tabular}

Table 2: Dimensions of online behaviour summary.

\begin{tabular}{lrr}
\hline Behaviour & Facebook & Instagram \\
\hline & $M(S D)$ & $M(S D)$ \\
Consumptive & $\mathbf{1 . 6 7 ( 1 . 4 6 )}$ & $\mathbf{3 . 5 7 ( \mathbf { 1 . 5 8 } )}$ \\
consume news feed & $3.02(2.07)$ & $4.27(1.76)$ \\
consume stories & $1.32(1.86)$ & $4.28(1.82)$ \\
consume live stories & $0.68(1.44)$ & $2.15(2.04)$ \\
Productive & $\mathbf{0 . 2 5 ( \mathbf { 0 . 4 5 } )}$ & $\mathbf{1 . 1 4 ( \mathbf { 0 . 9 3 } )}$ \\
Post status & $0.56(0.96)$ & $1.71(1.10)$ \\
Post stories & $0.16(0.57)$ & $1.50(1.54)$ \\
Post live stories & $0.02(0.22)$ & $0.21(0.79)$ \\
Reactive & $\mathbf{1 . 9 8 ( 1 . 5 7 )}$ & $2.71(\mathbf{1 . 3 3})$ \\
Liking posts & $2.77(1.92)$ & $4.50(1.70)$ \\
Commenting on posts & $1.83(1.84)$ & $2.67(1.84)$ \\
Sharing posts & $1.35(1.65)$ & $0.96(1.65)$ \\
\hline
\end{tabular}

in their second year, $52(20.00 \%)$ in their third year, $54(20.77 \%)$ in their fourth year, and $30(11.54 \%)$ had been studying for more than five years. While 166 participants $(64.62 \%)$ were funded by their parents or guardians, $82(31.54 \%)$ were funded by a bursary or scholarship, and only ten (3.85\%) were self-funded. A majority of participants $(51.92 \%)$ had a monthly social spending budget of between R0 and R1000, with only ten respondents (3.85\%) indicating a monthly social spending budget of R5000 or more.

\subsection{Online Behaviour}

Across the sample, 27 respondents (10.39\%) only had a Facebook account, 19 (7.31\%) only had an Instagram account, 195 (75.00\%) had both a Facebook and an Instagram account, and 19 (7.31\%) had neither. For the remaining analyses only those respondents $(n=241)$ who used at least one of the two SNSs were considered. Table 1 provides a summary of the daily use in hours for respondents who indicated that they used either Facebook, Instagram, or both platforms. For both SNSs the greatest proportion of respondents indicated that, on an average day, they use the platform for an hour. For Facebook, only 20 respondents $(8.30 \%)$ used the platform for three or more hours a day while, for Instagram, 55 respondents $(22.82 \%)$ indicated such use patterns. As indicated by a paired samples t-test, there was a small difference in the average reported daily use-duration between Instagram $(M=1.96, S D=1.06)$ and Facebook $(M=1.42, S D=0.82)$, with respondents reporting a greater amount of time on Instagram $(t(240)=-4.95, p<.001, d=-.32)$.

4.2.1 Productive Online Behaviour. Productive behaviour was assessed by analysing the responses for the three productive activities
- posting a status, posting a story, and posting a live story. As is evident in Table 2 productive behaviour on Facebook differed substantially across the three activities. Moreover, the results indicate that all three activities were rarely engaged in with the mean response falling between 0 and 1 (Never and a couple of times a year). In particular, for the posting of live stories only three respondents indicated that they performed this action at all. To derive a combined scale for productive behaviour on Facebook these three items were averaged and produced a scale (ranging from 0-6) with a mean of $0.25(S D=0.45)$. Given the relative differences in outcomes for the three activities, not unexpectedly, the scale demonstrated poor internal consistency (Cronbach's $\alpha=0.46$ ). As was the case with Facebook, for Instagram, differences existed between the three activities considered. Compared to statuses $(M=1.71, S D=1.10)$ or stories $(M=1.50, S D=1.54)$, the respondents rarely posted live stories $(M=0.21, S D=0.79)$. Averaging these three activities for Instagram produced a relatively internally consistent scale (Cronbach's $\alpha=0.69$ ) for productive behaviour on Instagram with a mean of $1.14(S D=0.93)$. A paired samples t-test indicated that, for productive behaviour, there was a large difference between the two platforms $(t(240)=-13.814, p<0.001, d=-0.889)$. Figure 1 provides a comparison of the productive behaviours on the SNSs. ${ }^{4}$

4.2.2 Consumptive Online Behaviour. Consumptive behaviour was assessed by analysing the responses for browsing the newsfeed, viewing stories, or viewing live stories. As with productive behaviour, an overall scale was calculated to represent a summary of consumptive behaviour on each platform (Facebook: Cronbach's $\alpha=$ 0.73; Instagram: Cronbach's $\alpha=0.80)$. As confirmed by a paired samples t-test $(t(240)=-11.714, p<0.001, d=-0.754)$, consumptive behaviour on Instagram $(3.57, S D=1.58)$ was reported to be more frequent than consumptive behaviour on Facebook $(M=1.67, S D=1.46)$, with a medium effect size. Figure 2 summarises the responses for each of the consumptive behaviours, for each platform.

4.2.3 Reactive Online Behaviour. To consider reactive online behaviour we analysed the responses for the three reactive behaviours considered -liking posts, commenting on posts, and sharing posts. Again, while differences existed between the various behaviours (summarised in Table 2), an overall scale for reactive online behaviour was calculated for each platform (Facebook: $\alpha=0.84$; Instagram: $\alpha=0.65$ ), with both indicating satisfactory internal consistency. As with productive and consumptive behaviours, the mean was greater for Instagram $(M=2.71, S D=1.33)$ than for Facebook $(M=1.98, S D=1.57)$. A paired samples t-test demonstrated that the effect size of this difference was small $(t(240)=$ $-4.700, p<.001, d=-0.303)$. Figure 3 indicates that, while some reactive behaviours occurred on Facebook, overall such behaviour was more common on Instagram.

\subsection{Online and Offline Social Behaviour}

To consider associations between online and offline social behaviour we first provide a descriptive summary of the results for offline behaviour, before presenting a correlational analysis.

\footnotetext{
${ }^{4}$ The $\mathrm{x}$-axis ranges from never (0), a couple of times a year (1), once a month (2), once a week (3), a couple of times a week (4), daily (5), and multiple times a day (6).
} 

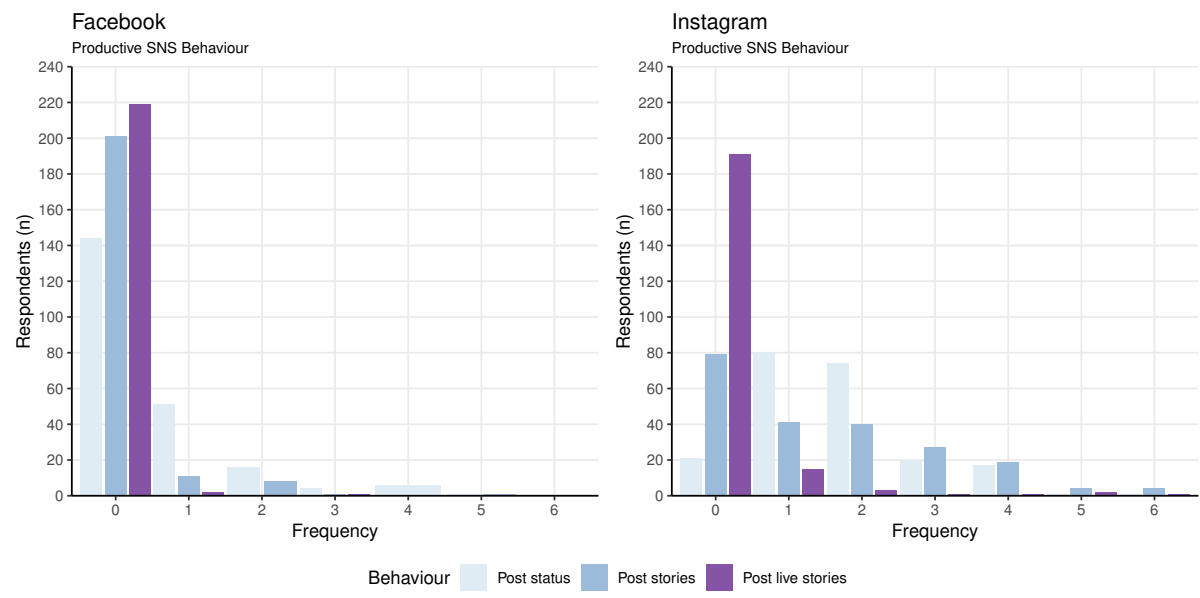

Figure 1: Productive behaviour on Facebook and Instagram.
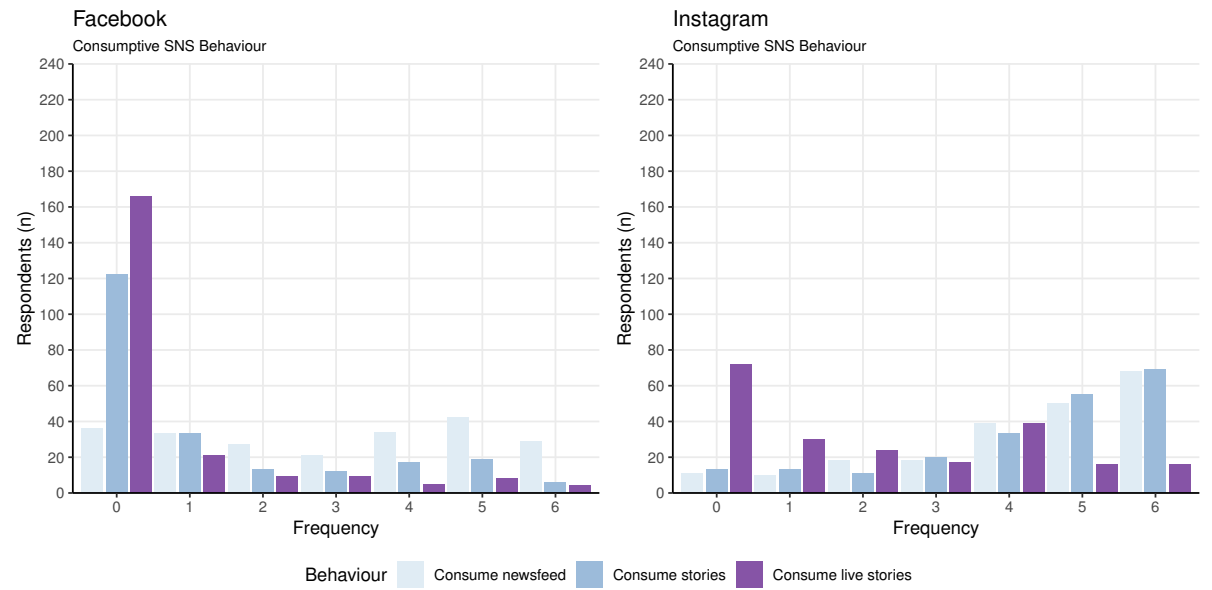

Figure 2: Consumptive behaviour on Facebook and Instagram.

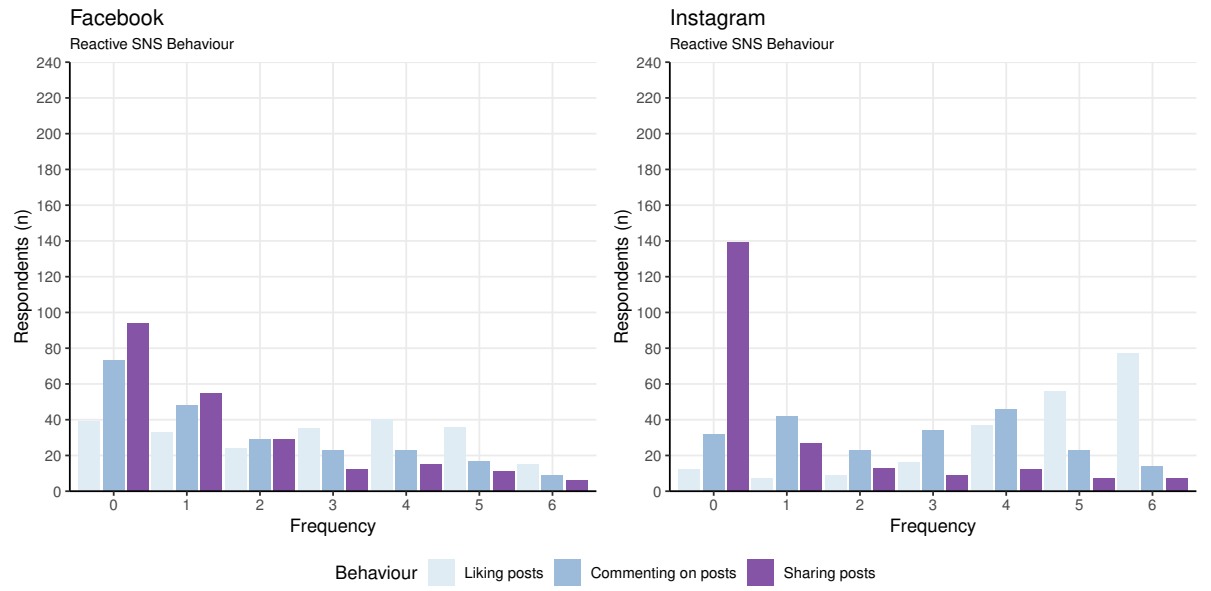

Figure 3: Reactive behaviour on Facebook and Instagram. 
Table 3: Summary of offline social behaviour responses.

\begin{tabular}{lrrrr}
\hline Frequency & \multicolumn{4}{c}{ Behaviour (\%) } \\
\hline & Groups <5 & Groups $>5$ & Family & Peers \\
\cline { 2 - 6 } Never & 0.41 & 2.48 & 0.83 & 2.49 \\
A couple times a year & 2.07 & 12.86 & 16.18 & 8.71 \\
Once a month & 3.32 & 22.41 & 13.69 & 13.28 \\
Once a week & 11.20 & 20.75 & 20.75 & 18.67 \\
A couple times a week & 20.75 & $\mathbf{2 3 . 6 5}$ & $\mathbf{2 2 . 8 2}$ & $\mathbf{2 4 . 4 8}$ \\
Daily & 30.71 & 12.86 & 15.77 & 19.92 \\
Multiple times a day & $\mathbf{3 1 . 5 4}$ & 4.98 & 9.96 & 12.45 \\
\hline
\end{tabular}

4.3.1 Offline Social Behaviour. Offline social behaviour was assessed by analysing the frequency with which the participants engaged in four categories of social behaviour -socialising in large groups (more than five people), socialising in small groups (less than five people), socialising with family members, and socialising with peers doing non-work related activities. Indicating the proportion of respondents who selected the various frequency options, Table 3 provides a summary of the results in this regard. For socialising in groups larger than five, with family, and with peers the largest proportion of respondents indicated that they engage in this activity a couple of times a week. While, for socialising in groups less than five, the largest proportion indicated that they engage in this activity multiple times a day.

4.3.2 Correlations Between Online and Offline Behaviour. To determine associations between the three categories of online social behaviour and the four categories of offline social behaviour, Spearman rank order correlation coefficients among the items were calculated (presented in Table 4). For Facebook, only reactive behaviour was shown to be associated with any form of offline behaviour. Specifically, socialising in groups of more than five individuals was positively associated with reactive actions on Facebook $\left(r_{s}=0.17, p<.05\right)$. For Instagram, all three online social behaviours were found to be positively associated with offline socialising in groups larger than five (consumptive: $r_{s}=0.23, p<.001$; productive: $r_{s}=0.27, p<.001$; reactive: $\left.r_{s}=0.29, p<.001\right)$. No associations between offline socialising with family members, or socialising in groups less than five, and online behaviour were found, while only a single association was found between offline socialising with peers and online behaviour - reacting on Instagram $\left(r_{s}=0.17, p<.05\right)$.

\subsection{Personality and Online Social Behaviour}

To consider possible effects of individual differences on online social behaviour a personality index was elicited from the respondents. This section begins with a description of these outcomes, before presenting a correlational analysis with online social behaviour.

4.4.1 Personality Outcomes. For each of the Big Five personality traits the respondents received a score ranging from four to 20 , with a higher score representing a greater prominence of the trait. Additionally, as is typical in personality research, to establish local norms, the scores for each individual were compared to the local mean derived from the sample to determine if their rating fell above
Table 4: Correlations between online and offline behaviour

\begin{tabular}{|c|c|c|c|c|}
\hline \multirow[t]{2}{*}{ Online Behaviour } & \multicolumn{4}{|c|}{ Offline Behaviour } \\
\hline & Groups $<5$ & Groups $>5$ & Family & Peers \\
\hline \multicolumn{5}{|l|}{ Facebook } \\
\hline Consumptive & -0.09 & 0.03 & -0.13 & -0.04 \\
\hline Productive & -0.07 & 0.01 & -0.11 & -0.07 \\
\hline Reactive & 0.01 & $0.17^{*}$ & -0.10 & 0.00 \\
\hline \multicolumn{5}{|l|}{ Instagram } \\
\hline Consumptive & 0.13 & $0.23^{* * *}$ & 0.08 & 0.08 \\
\hline Productive & 0.08 & $0.27^{* * *}$ & 0.02 & 0.09 \\
\hline Reactive & 0.14 & $0.29^{* * *}$ & 0.01 & $0.17^{*}$ \\
\hline
\end{tabular}

Table 5: Summary of Personality Trait Outcomes.

\begin{tabular}{lrrrrrr}
\hline Personality trait & \multicolumn{1}{c}{$M$} & $S D$ & $\langle M(\%)$ & $>M(\%)$ & skewness & kurtosis \\
\hline Extroversion & 11.69 & 3.84 & 48.55 & 51.45 & 0.01 & -0.58 \\
Agreeableness & 15.58 & 2.76 & 43.15 & 56.85 & -0.54 & -0.11 \\
Conscientiousness & 13.97 & 2.79 & 40.25 & 59.75 & -0.15 & -0.22 \\
Neuroticism & 12.05 & 3.17 & 58.09 & 41.91 & 0.10 & -0.15 \\
Openness & 15.47 & 2.69 & 49.38 & 50.62 & -0.23 & -0.19 \\
\hline
\end{tabular}

or below this mean. Table 5 provides a summary of the outcomes in this regard. Across the sample, scores for all five traits were approximately symmetric, falling in the acceptable range for normality for such scales, with the exception of agreeableness which was moderately skewed to the left. Such outcomes are in-line with norms for the mini-IPIP scale [e.g., 3, 14]. While, much variability existed in the mean outcome, for extroversion, agreeableness, conscientiousness, and openness a majority of respondents fell above the mean while, for neuroticism, the majority fell below the mean.

4.4.2 Associations Between Personality and Online Social Behaviour. To determine associations between the three categories of online social behaviour and the five personality traits, Pearson productmoment correlation coefficients among the items were calculated (presented in Table 6). For Facebook, reactive behaviour was found to be positively associated with extroversion $(r=0.14, p<.05)$ and neuroticism $(r=0.15, p<.05)$, while consumptive behaviour was negatively correlated with openness $(r=-0.18, p<.05)$. No associations were found for productive behaviour on Facebook. For Instagram, extroversion was found to be positively associated with consumptive $(r=0.19, p<.05)$, productive $(r=0.32, p<.001)$, and reactive $(r=0.27, p<.001)$ behaviour, while agreeableness was positively associated with productive $(r=0.20, p<.001)$ and reactive $(r=0.14, p<.05)$ behaviour. No associations between behaviour on Instagram and conscientiousness, neuroticism, or openness were found. Across both platforms, no associations between online social behaviour and conscientiousness were found.

\subsection{Comparative Analysis}

To assess the proportion of variance in offline social behaviour explained by online social behaviour, after accounting for personality traits, a series of hierarchically ordered multiple linear regression 
Table 6: Correlations between online behaviour and personality traits.

\begin{tabular}{|c|c|c|c|c|c|}
\hline \multirow[t]{2}{*}{ Online Behaviour } & \multicolumn{5}{|c|}{ Big Five Personality Trait } \\
\hline & Ext & Agr & Con & $\mathrm{Neu}$ & Ope \\
\hline \multicolumn{6}{|l|}{ Facebook } \\
\hline Consumptive & -0.02 & -0.05 & -0.01 & 0.07 & $-0.18^{*}$ \\
\hline Productive & -0.06 & 0.07 & 0.03 & 0.13 & 0.01 \\
\hline Reactive & $0.14^{*}$ & 0.12 & 0.07 & $0.15^{*}$ & -0.03 \\
\hline \multicolumn{6}{|l|}{ Instagram } \\
\hline Consumptive & $0.19^{*}$ & 0.04 & 0.04 & 0.08 & -0.06 \\
\hline Productive & $0.32^{* * *}$ & 0.20 *** & 0.14 & 0.11 & 0.02 \\
\hline Reactive & $0.27^{* * *}$ & $0.14^{*}$ & 0.07 & 0.07 & 0.01 \\
\hline
\end{tabular}

equations were fitted to the data. ${ }^{5}$ Considering the correlations depicted in Table 4, this analysis $(n=214)$ was only conducted for one platform (Instagram) and one form of offline social behaviour -socialising in groups larger than five. Prior to the analysis, it was determined that a sufficiently low level of multicollinearity was present (extroversion: $V I F=1.30$; agreeableness: $V I F=1.18$; conscientiousness: $V I F=1.07$; neuroticism: $V I F=1.10$; openness: $V I F=1.05 ;$ IN productive $: V I F=1.47 ;$ IN reactive $: V I F=2.13$; IN consumptive: $V I F=1.84$ ). Table 7 presents a summary of the outcomes of the hierarchical analysis. In the first step a linear regression model was fitted with socialising in groups larger than five as the response variable and the five personality traits as the explanatory variables $\left(R^{2}=0.280, F(5,208)=16.2, p<0.001\right)$. In the second step a linear regression model was fitted with the same response variable but with the three online behaviours on Instagram (reactive, productive, and consumptive) added as additional explanatory variables $\left(R^{2}=0.323, F(8,205), P<0.001\right)$. Consequently, the addition of the three online social behaviour variables explained an additional $4.3 \%$ of the variance in offline social behaviour $\left(\Delta R^{2}=0.043, F(3,205), p<0.01\right)$. Despite this, when considering the specific coefficients in the final model, only extroversion and neuroticism were significant predictors of offline social behaviour. Holding all other variables considered constant, increases in extroversion correspond to increased levels of offline socialisation in large groups, while increases in neuroticism correspond negatively with offline socialisation in large groups.

\section{DISCUSSION}

Building on studies indicating associations between online and offline behaviour [35], situated within Vodanovich et al. [45]'s agenda for IS research, an exploratory study was conducted to describe patterns of online social behaviour among digital natives on two popular SNSs (Facebook and Instagram). To this end, extending from previous research [10, 11, 47], a typology for behaviour on SNSs involving three categories - producing, consuming, and reacting-

\footnotetext{
${ }^{5}$ Due to indications that ordinal logistic analyses are not well suited to hierarchical analyses seeking to compare model fit, the categorical response variable was treated as a continuous variable. In a recent, well-cited analysis Rhemtulla et al. [34] provide evidence indicating that, when the sample considered is larger than 100 , and when there exist seven or more categories, treating such categorical variables as continuous is acceptable. No difference in Type I error rates and minimal differences in biases for parameter estimates occur.
}

Table 7: Hierarchically ordered regression analysis summary.

\begin{tabular}{|c|c|c|c|c|c|c|c|c|}
\hline \multirow[t]{2}{*}{ Variable } & \multicolumn{4}{|c|}{ Model 1} & \multicolumn{4}{|c|}{ Model 2} \\
\hline & B & SE & $\beta$ & $t$ & B & $\mathrm{SE}$ & $\beta$ & $t$ \\
\hline Extroversion & 0.18 & 0.02 & 0.467 & $7.22^{* * *}$ & 0.15 & 0.03 & 0.39 & $5.96^{* * *}$ \\
\hline Agreeableness & 0.05 & 0.03 & 0.10 & 1.58 & 0.05 & 0.03 & 0.09 & 1.51 \\
\hline Conscientiousness & 0.02 & 0.03 & 0.04 & 0.67 & 0.01 & 0.03 & 0.02 & 0.37 \\
\hline Neuroticism & -0.05 & 0.03 & -0.10 & -1.68 & -0.06 & 0.03 & -0.14 & $-2.24^{*}$ \\
\hline Openness & 0.01 & 0.03 & 0.02 & 0.35 & 0.02 & 0.03 & 0.04 & 0.61 \\
\hline IN Productive & & & & & 0.05 & 0.11 & 0.03 & 0.47 \\
\hline IN Reactive & & & & & 0.13 & 0.09 & 0.12 & 1.37 \\
\hline IN Consumptive & & & & & 0.10 & 0.07 & 0.11 & 1.35 \\
\hline$R^{2}$ & 0.280 & & & & 0.323 & & & \\
\hline $\operatorname{adj.} R^{2}$ & 0.263 & & & & 0.297 & & & \\
\hline F Statistic & $16.2^{* *}$ & ** (df & $=5 ; 208$ & & $12.22^{*}$ & $* * *(\mathrm{~d}$ & $=8 ; 2$ & 05) \\
\hline$\Delta R^{2}$ & & & & & $0.043^{*}$ & & & \\
\hline
\end{tabular}

was proposed. In addition to enabling comparisons between the platforms, this enabled the analysis of associations between these actions, offline social behaviour, and personality traits. Given the original typology adopted, the absence of studies indicating particular causal directions, the changing nature of online behaviour, and the novel measures used in the survey, an exploratory frame was adopted for this study. As such, the following discussion outlines tentative findings that are of particular interest for future study and proposes ways in which these exploratory results can be tested.

For online social behaviour, the findings are broadly commensurate with previous results indicating that, among the target population, Instagram is used more frequently than Facebook [30, 31]. While no longitudinal data were collected in the present study, this outcome is reflective of a global trend indicating that a declining proportion of digital natives are making use of Facebook [30]. Perrin [30], for instance, has shown that $44 \%$ of those aged between 18 and 29 in the United States have deleted Facebook from their smartphones in the last year.

For the three dimensions of SNS behaviour the findings indicate that, for both platforms, productive behaviour occurred the least frequently. For Instagram, consumptive behaviour occurred the most frequently while, for Facebook, reactive behaviour was engaged in most frequently by the respondents. Overall, across the two platforms, SNS behaviour was shown to be characterised, primarily, by passive, undirected consumption as opposed to active, direct engagement with other users. This finding corresponds to Gerson et al. [18] and to Nielsen [24]'s 90-9-1 rule, with a majority of users primarily engaging in consumptive behaviour and only a minority actively contributing. Moreover, given previous findings indicating that non-reciprocity-evoking behaviours have been associated adversely with psycho-social well-being [39, 44, 47], and the present finding that passive use was more prominent than active use, it is reasonable to propose that, among the present sample, use of SNSs may be negatively associated with psycho-social wellbeing. Further research is, however, required to determine if this is the case and, additionally, whether SNS behaviour plays a causal role in this relationship or if other individual factors are of primary importance. Additionally, it is necessary to consider why passive 
behaviour occurs more frequently than active behaviour on SNSs. This may be due to the affordances of specific SNSs, with other services engendering more instances of active use, or it may be due to the sociotechnical relationship that has emerged been SNSs and their users. In particular, from the perspective of the Uses and Gratifications theory, users may adopt a utilitarian approach to their engagements with SNSs and use them to gratify certain needs (i.e., information seeking vs. content creation) [38].

For RQ3, it was found that, for Facebook, only reactive behaviour was associated with any form of offline social behaviour. This outcome may be reflective of the declining use of the platform with reacting, a relatively 'lightweight' form of active use occurring more frequently than other forms of active, reciprocity-evoking behaviour. For instagram, on the other hand, all three behavioural dimensions were found to relate positively with offline socialising in groups larger than five. Additionally, reactive behaviour was positively associated with offline socialising with peers outside of work situations. For both platforms no associations between online behaviour and socialisation with family members or in groups of less than five individuals were found. A key outcome of this study is that, across platforms and online behavioural forms, no negative associations with offline social behaviour were found. While relationships were only shown for offline socialisation in groups larger than five and, for reactive behaviour on Instagram, socialisation with peers outside of academic settings, all associations were positive albeit small in magnitude. This indicates that, despite reports [41], online social behaviour is not associated with lower levels of offline socialisation. It is proposed that the different outcomes between the platforms are largely indicative of changing use patterns and a generational shift away from Facebook towards other SNSs.

For RQ4, while a number of specific associations were found, the strongest associations between online behaviour and personality traits existed between productive and reactive behaviour on Instagram and extroversion. This outcome is indicative of a tendency of extroverted individuals to actively seek out social interaction and supports Liu and Campbell [23] who found that extroversion was the strongest predictor of activity on SNSs. Across both platforms, no associations between online social behaviour and conscientiousness were found. The only negative association was observed between openness and consumptive behaviour on Facebook.

Extending these findings, it was shown that, after accounting for the effect of personality traits, online social behaviour on Instagram accounted for an additional $4.3 \%$ of the variance in offline socialising in groups larger than five. Despite finding that online social behaviour provides additional explanatory power over offline social behaviour, it is important to note, firstly, that the increase was small, secondly, that personality traits accounted for a much larger proportion of the variance than online social behavior, thirdly, that this analysis only considered the offline behaviours for which a consistent correlation was shown and, finally, that behaviour on only a single platform was analysed. Considering specific personality traits, extroversion was seen to correspond with increased levels of offline socialisation in large groups, while increases in neuroticism were found to negatively predict such behaviour.

\subsection{Limitations and Recommendations}

While this study has generated novel findings which merit further investigation, a number of limitations exist. The first relates to the research design adopted. As an exploratory study the findings should be interpreted as preliminary indications of associations and effects, with subsequent confirmatory research needed to replicate and test the extent to which the results hold up to further examination. Additionally, given the cross-sectional and correlational design, no causal claims are provided. For this, further research adopting longitudinal or experimental designs is necessary. Further limitations relate to the assessment of online social behaviour. While the platforms considered were selected based on their widespread popularity, only assessing behaviour on two platforms does imply that the extent to which the findings can be generalised to other forms of online social behaviour is limited. In addition to this, given poor associations between objective measures of media use and retrospective self-report measures [7], the manner in which use behaviour was assessed presents as a limitation. Future studies should endeavour to assess both online and offline behaviour through objective means. Finally, because a singular population was targeted, the extent to which the present findings would generalise to non-student populations is unknown.

In addition to the suggestions for future research already provided, it is recommended that studies adopting a confirmatory framework, with larger and more diverse samples, be conducted to test the findings described. Moreover, it is recommended that the psychometric properties and construct validity of the scales proposed for the typology of SNS behaviour be assessed. Finally, to extend the present findings, studies should adopt an interpretivist epistemology and consider the collection of qualitative data to better understand the motivations for particular online social actions as well as perceptions of interactions with offline social behaviour.

\section{CONCLUSION}

Notwithstanding the aforementioned limitations, this exploratory study has provided a number of important contributions to research in this domain. First, it is argued that the simple typology presented in this study - reactive, consumptive, and productive- is a useful simplification of behaviour on SNSs. Second, while a positive association was found between some forms of online social behaviour and some forms of offline behaviour, it was shown, firstly, that this is not the case for all forms of social behaviour and, secondly, that this relationship differs depending on platform and platform-actions. Third, the study has shown that, after accounting for personality traits, an increase in explanatory power over some forms of offline social behaviour is provided by considering online social behaviour. Despite this, as was evident by both the correlational and regression analyses, personality traits account for a larger proportion of the variance in offline social behaviour than online social behaviour. Extroversion, in particular, was seen to be a particularly substantive predictor of offline social behaviour. The findings presented provide a useful point of departure for further study into associations between behaviour online and behaviour offline. 


\section{REFERENCES}

[1] Dohyun Ahn and Dong-Hee Shin. 2013. Is the social use of media for seeking connectedness or for avoiding social isolation? Mechanisms underlying media use and subjective well-being. Computers in Human Behavior 29, 6 (2013), 2453 2462. https://doi.org/10.1016/j.chb.2012.12.022

[2] Yoram Bachrach, Michal Kosinski, Thore Graepel, Pushmeet Kohli, and David Stillwell. 2012. Personality and Patterns of Facebook Usage. In Proceedings of the 4th Annual ACM Web Science Conference (WebSci '12). 24-32. https://doi.org/10 $1145 / 2380718.2380722$

[3] Ruth E. Baldasaro, Michael J. Shanahan, and Daniel J. Bauer. 2013. Psychometric properties of the mini-IPIP in a large, nationally representative sample of young adults. Fournal of Personality Assessment 95, 1 (2013), 74-84. https://doi.org/10. 1080/00223891.2012.700466

[4] Alixandra Barasch and Jonah Berger. 2014. Broadcasting and narrowcasting How audience size affects what people share. Journal of Marketing Research 51, 3 (2014), 286-299.

[5] Roy Baumeister and Mark Leary. 1995. The need To belong: Desire for interpersonal attachments as a fundamental human-motivation. Psychological Bulletin 117, 3 (1995), 497-529.

[6] Natalya N Bazarova. 2012. Public intimacy: Disclosure interpretation and social judgments on Facebook. fournal of Communication 62, 5 (2012), 815-832.

[7] Jeffrey Boase and Rich Ling. 2013. Measuring Mobile Phone Use: Self-Report versus Log Data. Journal of Computer-Mediated Communication 18, 4 (2013) 508-519. https://doi.org/10.1111/jcc4.12021

[8] Angela Bohn, Christian Buchta, Kurt Hornik, and Patrick Mair. 2014. Making friends and communicating on Facebook: Implications for the access to social capital. Social Networks 37 (2014), 29 - 41. https://doi.org/10.1016/j.socnet.2013. 11.003

[9] dana boyd and Nicole Ellison. 2007. Social network sites: Definition, history, and scholarship. Journal of computer-mediated Communication 13, 1 (2007), 210-230.

[10] Petter Brandtzaeg and Jan Heim. 2011. A typology of social networking sites users. International fournal of Web Based Communities 7, 1 (2011), 28-51.

[11] Moira Burke, Cameron Marlow, and Thomas Lento. 2010. Social Network Activity and Social Well-being. In Proceedings of the SIGCHI Conference on Human Factors in Computing Systems (CHI '10). ACM, New York, NY, USA, 1909-1912. https: //doi.org/10.1145/1753326.1753613

[12] Salvatore A. Catanese, Pasquale De Meo, Emilio Ferrara, Giacomo Fiumara, and Alessandro Provetti. 2011. Crawling Facebook for Social Network Analysis Purposes. , Article 52 (2011), 8 pages. https://doi.org/10.1145/1988688.1988749

[13] Wen-Bin Chiou, Szu-Wei Chen, and Da-Chi Liao. 2014. Does Facebook promote self-interest? Enactment of indiscriminate one-to-many communication on online social networking sites decreases prosocial behavior. Cyberpsychology, Behavior and Social Networking 17, 2 (2014), 68-73.

[14] Andrew Cooper, Luke Smillie, and Philip Corr. 2010. A confirmatory factor analysis of the Mini-IPIP five-factor model personality scale. Personality and Individual Differences 48, 5 (2010), 688-691. https://doi.org/10.1016/j.paid.2010. 01.004

[15] Fenne Große Deters and Matthias R Mehl. 2013. Does posting Facebook status updates increase or decrease loneliness? An online social networking experiment Social psychological and personality science 4, 5 (2013), 579-586.

[16] Brent Donnellan, Frederick L Oswald, Brendan M Baird, and Richard E Lucas. 2006. The mini-IPIP scales: tiny-yet-effective measures of the Big Five factors of personality. Psychological assessment 18, 2 (2006), 192.

[17] Mustafa Eşkisu, Rumeysa Hoşoğlu, and Kyler Rasmussen. 2017. An investigation of the relationship between Facebook usage, Big Five, self-esteem and narcissism. Computers in Human Behavior 69 (2017), 294-301.

[18] Jennifer Gerson, Anke C. Plagnol, and Philip J. Corr. 2017. Passive and Active Facebook Use Measure (PAUM): Validation and relationship to the Reinforcement Sensitivity Theory. Personality and Individual Differences 117 (2017), 81 - 90 https://doi.org/10.1016/j.paid.2017.05.034

[19] Anthony Giddens. 1979. Agency, Structure. Macmillan Education UK, London. https://doi.org/10.1007/978-1-349-16161-4_3

[20] Eric Gilbert and Karrie Karahalios. 2009. Predicting Tie Strength with Social Media. In Proceedings of the SIGCHI Conference on Human Factors in Computing Systems (CHI '09). ACM, New York, NY, USA, 211-220. https://doi.org/10.1145/ 1518701.1518736

[21] Rachel Grieve, Michaelle Indian, Kate Witteveen, G. Anne Tolan, and Jessica Marrington. 2013. Face-to-face or Facebook: Can social connectedness be derived online? Computers in Human Behavior 29, 3 (2013), 604 - 609. https://doi.org/10. 1016/j.chb.2012.11.017

[22] Yuheng Hu, Lydia Manikonda, Subbarao Kambhampati, et al. 2014. What We Instagram: A First Analysis of Instagram Photo Content and User Types. (2014).

[23] Dong Liu and W. Keith Campbell. 2017. The Big Five personality traits, Big Two metatraits and social media: A meta-analysis. Fournal of Research in Personality 70 (2017), 229 - 240. https://doi.org/10.1016/j.jrp.2017.08.004

[24] Jakob Nielsen. 2006. The 90-9-1 Rule for Participation Inequality in Social Media and Online Communities.
[25] M. Nowak and G. Spiller. 2017. Two Billion People Coming Together on Facebook. https://newsroom.fb.com/news/2017/06/ two-billion-people-coming-together-on-facebook/ Accessed 27.03.2019.

[26] Hyun Jung Oh, Elif Ozkaya, and Robert Larose. 2014. How does online social networking enhance life satisfaction? the relationships among online supportive interaction, affect, perceived social support, sense of community, and life satisfaction. Computers in Human Behavior 30 (2014), 69-78. https: //doi.org/10.1016/j.chb.2013.07.053 arXiv:0022-2429

[27] Amy C. Orben and Robin I.M. Dunbar. 2017. Social media and relationship development: The effect of valence and intimacy of posts. Computers in Human Behavior 73 (2017), 489 - 498. https://doi.org/10.1016/j.chb.2017.04.006

[28] Douglas A. Parry. 2017. The Digitally-Mediated Study Experiences of Undergraduate Students in South Africa. Master's Thesis. Stellenbosch University.

[29] Douglas A. Parry and Daniel B. le Roux. 2019. Off-Task Media Use in Lectures: Towards a Theory of Determinants. In ICT Education, Salah Kabanda, Hussein Suleman, and Stefan Gruner (Eds.). Springer International, Cham, 49-64.

[30] Andrew Perrin. 2018. Many US Facebook users have changed privacy settings or taken a break. http://www.pewresearch.org/fact-tank/2018/09/05/ americans-are-changing-their-relationship-with-facebook/

[31] Pew Research Center. 2018. Social Media Use in 2018. Technical Report.

[32] Thomas V Pollet, Sam GB Roberts, and Robin IM Dunbar. 2011. Use of social network sites and instant messaging does not lead to increased offline social network size, or to emotionally closer relationships with offline network members. Cyberpsychology, Behavior, and Social Networking 14, 4 (2011), 253-258.

[33] Marc Prensky. 2001. Digital Natives, Digital Immigrants Part 1. On the Horizon Journal of Service Management 9, 3 (2001), 245-267. https://doi.org/10.1108/ 10748120110424816

[34] Mijke Rhemtulla, Patricia E Brosseau-Liard, and Victoria Savalei. 2012. When can categorical variables be treated as continuous? A comparison of robust continuous and categorical SEM estimation methods under suboptimal conditions. Psychological methods 17, 3 (2012), 354.

[35] Tracii Ryan, Kelly A. Allen, De Leon L. Gray, and Dennis M. McInerney. 2017. How Social Are Social Media? A Review of Online Social Behaviour and Connectedness. Journal of Relationships Research (2017). https://doi.org/10.1017/jrr.2017.13

[36] William H. Sewell. 1992. A Theory of Structure: Duality, Agency, and Transformation. Amer. 7. Sociology 98, 1 (1992), 1-29. https://doi.org/10.1086/229967

[37] Kennon M Sheldon, Neetu Abad, and Christian Hinsch. 2011. A two-process view of Facebook use and relatedness need-satisfaction: Disconnection drives use, and connection rewards it. Journal of personality and social psychology 100 , 4 (2011), 766.

[38] Andrew D. Smock, Nicole B. Ellison, Cliff Lampe, and Donghee Yvette Wohn. 2011. Facebook as a toolkit: A uses and gratification approach to unbundling feature use. Computers in Human Behavior 27, 6 (2011), 2322-2329. https: //doi.org/10.1016/j.chb.2011.07.011

[39] Edson C Tandoc, Patrick Ferrucci, and Margaret Duffy. 2015. Facebook use, envy, and depression among college students: Is facebooking depressing? Computers in Human Behavior 43 (2015), 139-146.

[40] Eveline Teppers, Koen Luyckx, Theo A. Klimstra, and Luc Goossens. 2014. Loneliness and Facebook motives in adolescence: A longitudinal inquiry into directionality of effect. Fournal of Adolescence 37, 5 (2014), 691 - 699. https: //doi.org/10.1016/j.adolescence.2013.11.003

[41] Sherry Turkle. 2017. Alone together: Why we expect more from technology and less from each other. Hachette UK.

[42] Patti M. Valkenburg and Jochen Peter. 2007. Online Communication and Adolescent Well-Being: Testing the Stimulation Versus the Displacement Hypothesis. Fournal of Computer-Mediated Communication 12, 4 (2007), 1169-1182. https://doi.org/10.1111/j.1083-6101.2007.00368.x

[43] Patti M. Valkenburg, Sindy R. Sumter, and Jochen Peter. 2011. Gender differences in online and offline self-disclosure in pre-adolescence and adolescence. British Journal of Developmental Psychology 29, 2 (2011), 253-269. https://doi.org/10. 1348/2044-835X.002001

[44] Philippe Verduyn, David Seungjae Lee, Jiyoung Park, Holly Shablack, Ariana Orvell, Joseph Bayer, Oscar Ybarra, John Jonides, and Ethan Kross. 2015. Passive Facebook usage undermines affective well-being: Experimental and longitudinal evidence. Fournal of Experimental Psychology: General 144, 2 (2015), 480.

[45] Shahper Vodanovich, David Sundaram, and Michael Myers. 2010. Research Commentary: Digital natives and Ubiquitous Information Systems. Information Systems Research 21, 4 (2010), 711-723.

[46] Eric-Jan Wagenmakers, Ruud Wetzels, Denny Borsboom, Han L. J. van der Maas, and Rogier A. Kievit. 2012. An Agenda for Purely Confirmatory Research. Perspectives on Psychological Science 7, 6 (2012), 632-638.

[47] Helena Wenninger, Hanna Krasnova, and Peter Buxmann. 2018. Understanding the role of social networking sites in the subjective well-being of users: a diary study. European Journal of Information Systems (2018), 1-23.

[48] World Wide Worx. 2018. Social Media Landscape 2019 report. Technical Report. World Wide Worx. 